\title{
$\psi$-Exponential Stability of Nonlinear Impulsive Dynamic Equations on Time Scales
}

\author{
Veysel Fuat Hatipoğlu, ${ }^{1}$ Deniz Uçar, ${ }^{2}$ and Zeynep Fidan Koçak ${ }^{1}$ \\ ${ }^{1}$ Department of Mathematics, Faculty of Science, Muğla University, Kötekli Campus, \\ 48000 Muğla, Turkey \\ ${ }^{2}$ Department of Mathematics, Faculty of Sciences and Arts, Usak University, 1 Eylul Campus, \\ 64200 Usak, Turkey
}

Correspondence should be addressed to Veysel Fuat Hatipoğlu; veyselfuat.hatipoglu@mu.edu.tr

Received 26 November 2012; Accepted 15 March 2013

Academic Editor: Stefan Siegmund

Copyright (C) 2013 Veysel Fuat Hatipoğlu et al. This is an open access article distributed under the Creative Commons Attribution License, which permits unrestricted use, distribution, and reproduction in any medium, provided the original work is properly cited.

The purpose of this paper is to present the sufficient $\psi$-exponential, uniform exponential, and global exponential stability conditions for nonlinear impulsive dynamic systems on time scales.

\section{Introduction}

In recent years, a significant progress has been made in the stability theory of impulsive systems [1,2], and in [3] authors studied the $\psi$-exponential stability for nonlinear impulsive differential equations. There are various types of stability of dynamic systems on time scales such as asymptotic stability $[4,5]$, exponential and uniform exponential stability [6-8], and $h$-stability [9]. In the past decade, many authors studied impulsive dynamic systems on time scales [10-14]. There are some papers on the theory of the stability of impulsive dynamic systems on time scales. In [15], stability criteria for impulsive systems are given and in [16], authors studied $\psi$ uniform stability of linear impulsive dynamic systems.

In this paper, we consider the $\psi$-exponential stability of the zero solution of the first-order nonlinear impulsive dynamic system

$$
\begin{gathered}
x^{\Delta}(t)=f(t, x(t)), \quad t \in \mathbb{T}_{t_{0}}^{+}, t \neq t_{k}, \\
x\left(t_{k}^{+}\right)-x\left(t_{k}^{-}\right)=I_{k}\left(x\left(t_{k}^{-}\right)\right), \quad t=t_{k}, \quad k=1,2, \ldots, n, \\
x\left(t_{0}^{+}\right)=x_{0},
\end{gathered}
$$

where $\mathbb{T}$ is a time scale which has at least finitely many rightdense points of impulsive $t_{k}, f:[0, \infty) \times \mathbb{R}^{n} \rightarrow \mathbb{R}^{n}$ is a nonlinear function and rd continuous in $\left(t_{k-1}, t_{k}\right] \times \mathbb{R}^{n}$, $I_{k} \in C_{\mathrm{rd}}\left[\mathbb{R}^{n}, \mathbb{R}^{n}\right]$, and $0 \leq t_{0}<t_{1}<t_{2}<\cdots<t_{n}<t$ are fixed moments of impulsive effect. Let $\psi_{i}: \mathbb{T} \rightarrow(0, \infty)$, $i=1,2, \ldots, n$, be rd continuous functions and let $\psi=$ $\operatorname{diag}\left[\psi_{1}, \psi_{2}, \ldots, \psi_{n}\right]$. Throughout the paper, we assume that $f(t, 0)=0$, for all $t$ in the time scale interval $[0, \infty)$, and call the zero function the trivial solution of (1) and we consider $\mathbb{W}_{t_{0}}^{+}=\left\{t \in \mathbb{T}: t \geq t_{0}\right\}$. Existence and uniqueness of solutions of (1) have been studied in [10].

In the following part we present some basic concepts about time scale calculus and we refer the reader to resource [17] for more detailed information on dynamic equations on time scales.

\section{Preliminaries}

A time scale $\mathbb{T}$ is an arbitrary nonempty closed subset of the real numbers $\mathbb{R}$. For $t \in \mathbb{T}$ we define the forward jump operator $\sigma: \mathbb{T} \rightarrow \mathbb{T}$ by

$$
\sigma(t):=\inf \{s \in \mathbb{T}: s>t\}
$$

while the backward jump operator $\rho: \mathbb{T} \rightarrow \mathbb{T}$ is defined by

$$
\rho(t):=\sup \{s \in \mathbb{T}: s<t\} \text {. }
$$


If $\sigma(t)>t$, we say that $t$ is right scattered, while if $\rho(t)<t$, we say that $t$ is left scattered. Also, if $\sigma(t)=t$, then $t$ is called right dense, and if $\rho(t)=t$, then $t$ is called left dense. The graininess function $\mu: \mathbb{T} \rightarrow[0, \infty)$ is defined by

$$
\mu(t):=\sigma(t)-t
$$

We introduce the set $\mathbb{T}^{\mathcal{K}}$ which is derived from the time scale $\mathbb{T}$ as follows. If $\mathbb{T}$ has a left-scattered maximum $m$, then $\mathbb{T}^{\mathcal{K}}=\mathbb{T}-\{m\}$; otherwise $\mathbb{T}^{\mathcal{K}}=\mathbb{T}$.

A function $f$ on $\mathbb{T}$ is said to be delta differentiable at some point $t \in \mathbb{T}$ if there is a number $f^{\Delta}(t)$ such that for every $\varepsilon>0$ there is a neighborhood $U \subset \mathbb{T}$ of $t$ such that

$$
\begin{array}{r}
\left|f(\sigma(t))-f(s)-f^{\Delta}(t)(\sigma(t)-s)\right| \leq \varepsilon|\sigma(t)-s|, \\
s \in U .
\end{array}
$$

The function $p: \mathbb{T} \rightarrow \mathbb{R}$ is said to be regressive provided $1+\mu(t) p(t) \neq 0$ for all $t \in \mathbb{T}^{\kappa}$. The set of all regressive rdcontinuous functions $f: \mathbb{T} \rightarrow \mathbb{R}$ is denoted by $\mathfrak{R}$.

Let $p \in \mathfrak{R}$ and $\mu(t) \neq 0$ for all $t \in \mathbb{T}$. The exponential function on $\mathbb{T}$, defined by

$$
e_{p}(t, s)=\exp \left(\int_{s}^{t} \frac{1}{\mu(z)} \log (1+\mu(z) p(z)) \Delta z\right),
$$

is the solution to the initial value problem $y^{\Delta}=p(t) y$, $y(s)=1$. Properties of the exponential function on $\mathbb{T}$ are given in [6].

In [6] authors defined the Lyapunov function on time scales, type I Lyapunov function $V$ as,

$$
V(x)=\sum_{i=1}^{n} V_{i}\left(x_{i}\right)=V_{1}\left(x_{1}\right)+\cdots+V_{n}\left(x_{n}\right),
$$

and $\Delta$ derivative of type I Lyapunov function as follows:

$$
\begin{aligned}
& {[V(x(t))]^{\Delta}} \\
& \quad= \begin{cases}\sum_{i=1}^{n} \frac{\left[V_{i}\left(x_{i}+\mu(t) f_{i}(t, x)\right)-V_{i}\left(x_{i}\right)\right]}{\mu(t)} & \text { for } \mu(t) \neq 0, \\
\nabla V(x) \cdot f(t, x) & \text { for } \mu(t)=0 .\end{cases}
\end{aligned}
$$

We start introducing notations that will be used in the following sections. In the Euclidean $n$-space, norm of a vector $x=\left\{x_{1}, x_{2}, \ldots, x_{n}\right\}^{T}$ is given by $\|x\|=$ $\max \left\{\left|x_{1}\right|,\left|x_{2}\right|, \ldots,\left|x_{n}\right|\right\}$. The induced norm of an $n \times n$ matrix $A$ is defined to be $\|A\|=\sup _{\|x\| \leq 1}\|A x\|$.

Now, we give definition of $\psi$-exponential, $\psi$-uniform exponential, $\psi$-global exponential stability, and stability conditions for the solution of nonlinear impulsive dynamic system (1).

\section{3. $\psi$-Exponential Stability}

Definition 1. The trivial solution to (1) is $\psi$ exponentially stable on $[0, \infty)$ if any solution $x\left(t, t_{0}, x_{0}\right)$ of the system (1) satisfies for all $t \in\left[t_{k-1}, t_{k}\right), k=1,2, \ldots, n$,

$$
\left\|\psi(t) x\left(t, t_{0}, x_{0}\right)\right\| \leq C\left(\left\|x_{0}\right\|, t_{0}\right)\left(e_{\ominus M}\left(t, t_{0}\right)\right)^{d},
$$

where $d$ is a positive constant and $C(h, t) \in \mathbb{R}^{+} \times \mathbb{T}_{t_{0}}^{+} \rightarrow \mathbb{R}^{+}$ is a nonnegative increasing function, $M>0$. If the function $C$ is independent of $t_{0}$, then the trivial solution to system (1) is said to be $\psi$ uniformly exponentially stable on $[0, \infty)$.

Definition 2. The trivial solution to (1) is $\psi$ globally exponentially stable on $[0, \infty)$ if there exist some constants $\delta>0$ and $M \geq 1$ such that any solution $x\left(t, t_{0}, x_{0}\right)$ of (1), for all $t \in\left[t_{k-1}, t_{k}\right), k=1,2, \ldots, n$, we have

$$
\left\|\psi(t) x\left(t, t_{0}, x_{0}\right)\right\| \leq M e_{\ominus \delta}\left(t, t_{0}\right) .
$$

Now, we shall present sufficient conditions for the $\psi$ exponential stability, $\psi$ uniformly exponential stability, and $\psi$ globally exponentially stability of(1).

Theorem 3. Assume that $D \subset \mathbb{R}^{n}$ contains the origin and there exists a type I Lyapunov function $V: \mathbb{T}_{t_{0}}^{+} \times D \rightarrow[0, \infty)$ such that, for all $(t, x) \in \mathbb{T}_{t_{0}}^{+} \times D$ and $t \in\left[t_{k-1}, t_{k}\right), k=1,2, \ldots, n$,

$$
\begin{gathered}
\lambda_{1}(t)\|\psi(t) x(t)\|^{p} \leq V(t, x) \leq \lambda_{2}(t)\|\psi(t) x(t)\|^{q} \\
V^{\Delta}(t, x) \leq \frac{-\lambda_{3}(t)\|\psi(t) x(t)\|^{r}-L(M \ominus \delta) e_{\ominus \delta}\left(t, t_{0}\right)}{1+M \mu(t)} \\
V(t, x)-V^{r / q}(t, x) \leq \gamma e_{\ominus \delta}\left(t, t_{0}\right)
\end{gathered}
$$

where $\lambda_{1}(t), \lambda_{2}(t)$, and $\lambda_{3}(t)$ are positive functions, where $\lambda_{1}(t)$ is nondecreasing; $p, q, r$, and $\gamma$ are positive constants; $L$ is a nonnegative constant, and $\delta>M:=\inf _{t \geq 0} \lambda_{3}(t) /\left[\lambda_{2}(t)\right]^{r / q}>$ 0 . Then the trivial solution to (1) is $\psi$ exponentially stable on $[0, \infty)$.

Proof. Let $x$ be a solution to (1) that stays in $D$ for all $t \geq t_{0}$. As $M:=\inf _{t \geq 0} \lambda_{3}(t) /\left[\lambda_{2}(t)\right]^{r / q}>0, e_{M}\left(t, t_{0}\right)$ is well defined and positive. Thus $\lambda_{3}(t) /\left[\lambda_{2}(t)\right]^{r / q} \geq M$. Consider

$$
\begin{aligned}
{[V(} & \left.t, x(t)) e_{M}\left(t, t_{0}\right)\right]^{\Delta} \\
= & V^{\Delta}(t, x(t)) e_{M}^{\sigma}\left(t, t_{0}\right)+V(t, x(t)) e_{M}^{\Delta}\left(t, t_{0}\right), \\
\leq & \left(-\lambda_{3}(t)\|\psi(t) x(t)\|^{r}-L(M \ominus \delta) e_{\ominus \delta}\left(t, t_{0}\right)\right) e_{M}\left(t, t_{0}\right) \\
& +M V(t, x(t)) e_{M}\left(t, t_{0}\right) \\
= & \left(-\lambda_{3}(t)\|\psi(t) x(t)\|^{r}+M V(t, x(t))-L(M \ominus \delta) e_{\ominus \delta}\left(t, t_{0}\right)\right) \\
& \times e_{M}\left(t, t_{0}\right) \\
\leq & \left(\frac{-\lambda_{3}(t)}{\left[\lambda_{2}(t)\right]^{r / q}} V^{r / q}(t, x(t))+M V(t, x(t))\right. \\
& \left.\quad-L(M \ominus \delta) e_{\ominus \delta}\left(t, t_{0}\right)\right) e_{M}\left(t, t_{0}\right)
\end{aligned}
$$




$$
\begin{aligned}
\leq & \left(M\left(V(t, x(t))-V^{r / q}(t, x(t))\right)-L(M \ominus \delta) e_{\ominus \delta}\left(t, t_{0}\right)\right) \\
& \times e_{M}\left(t, t_{0}\right) \\
\leq & (M \gamma-L(M \ominus \delta)) e_{M \ominus \delta}\left(t, t_{0}\right) .
\end{aligned}
$$

Integrating both sides of above inequality from $t_{0}$ to $t$ with $x_{0}=x\left(t_{0}\right)$, we obtain, for $t \in\left[t_{k-1}, t_{k}\right)$,

$$
\begin{aligned}
V(t, x) e_{M}\left(t, t_{0}\right) \leq & V\left(t_{0}, x_{0}\right) \\
& +\int_{t_{0}}^{t}(M \gamma-L(M \ominus \delta)) e_{M \ominus \delta}\left(\tau, t_{0}\right) \Delta \tau \\
= & V\left(t_{0}, x_{0}\right)+\left(\frac{M \gamma}{M \ominus \delta}-L\right) e_{M \ominus \delta}\left(t, t_{0}\right) \\
& +\frac{M \gamma}{\delta \ominus M}+L \\
\leq & V\left(t_{0}, x_{0}\right)+\frac{M \gamma}{\delta \ominus M}+L .
\end{aligned}
$$

From condition $V\left(t_{0}, x_{0}\right) \leq \lambda_{2}\left(t_{0}\right)\left\|\psi\left(t_{0}\right) x_{0}\right\|^{q}$

$$
V(t, x) e_{M}\left(t, t_{0}\right) \leq \lambda_{2}\left(t_{0}\right)\left\|\psi\left(t_{0}\right) x_{0}\right\|^{q}+\frac{M \gamma}{\delta \ominus M}+L .
$$

Letting

$$
\lambda_{2}\left(t_{0}\right)\left\|\psi\left(t_{0}\right) x_{0}\right\|^{q}+\frac{M \gamma}{\delta \ominus M}+L=C\left(\left\|x_{0}\right\|, t_{0}\right)>0
$$

we get,

$$
V(t, x) e_{M}\left(t, t_{0}\right) \leq C\left(\left\|x_{0}\right\|, t_{0}\right) .
$$

By condition (11), we have

$$
\|\psi(t) x(t)\| \leq \lambda_{1}^{-1 / p}(t)(V(t, x))^{1 / p}
$$

And by the fact that $\lambda_{1}(t) \geq \lambda_{1}\left(t_{0}\right)$, we obtain

$$
\|\psi(t) x(t)\| \leq \lambda_{1}^{-1 / p}\left(t_{0}\right)(V(t, x))^{1 / p} .
$$

From (18) and (20) we obtain the result for all, $t \in\left[t_{k-1}, t_{k}\right.$ ), $k=1,2, \ldots, n$,

$$
\|\psi(t) x(t)\| \leq \lambda_{1}^{-1 / p}\left(t_{0}\right)\left(C\left(\left\|x_{0}\right\|, t_{0}\right)\right)^{1 / p} e_{\ominus M}\left(t, t_{0}\right)^{1 / p} .
$$

By Definition 1 system (1) is $\psi$ exponentially stable.

If we consider $\psi$ as scaler function independent of $t$, then we get a sufficient condition for $\psi$ uniformly exponential stability as stated below.

Theorem 4. In Theorem 3 if $\psi$ is a constant function independent of $t$ and $\lambda_{i}(t)=\lambda_{i}, i=1,2,3$, are positive constants, then the trivial solution to system (1) is $\psi$ uniformly exponentially stable on $[0, \infty)$.
Proof. The proof is similar to proof of Theorem 3 by taking $\delta>\lambda_{3} /\left[\lambda_{2}\right]^{r / q}$ and $M=\lambda_{3} /\left[\lambda_{2}\right]^{r / q}$, hence omitted.

Theorem 5. Assume that $D \subset \mathbb{R}^{n}$ contains the origin and there exists a type I Lyapunov function $V: \mathbb{T}_{t_{0}}^{+} \times D \rightarrow[0, \infty)$ such that, for all $(t, x) \in \mathbb{T}_{t_{0}}^{+} \times D$ and $t \in\left[t_{k-1}, t_{k}\right), k=1,2, \ldots, n$,

$$
\begin{gathered}
\lambda_{1}\|\psi x(t)\|^{p} \leq V(x), \\
V^{\Delta}(t, x) \leq \frac{-\lambda_{2} V(x)-L(M \ominus \delta) e_{\ominus \delta}(t, 0)}{1+M \mu(t)},
\end{gathered}
$$

where $\psi$ is a constant function independent of $t . \lambda_{1}, \lambda_{2}, p, \delta>$ $0, L \geq 0$ are constants and $0<M<\min \left\{\lambda_{2}, \delta\right\}$. Then the trivial solution to (1) is $\psi$ uniformly exponentially stable on $[0, \infty)$.

Proof. Let $x$ be a solution to (1) that stays in $D$ for all $t \geq$ $t_{0}$. Since $M \in \mathfrak{R}^{+}, e_{M}(t, 0)$ is well defined and positive. Now consider

$$
\begin{aligned}
& {\left[V(x(t)) e_{M}(t, 0)\right]^{\Delta} } \\
&=V^{\Delta}(t, x(t)) e_{M}^{\sigma}(t, 0)+M V(x(t)) e_{M}(t, 0), \\
& \leq\left(-\lambda_{2} V(x(t))-L(M \ominus \delta) e_{\ominus \delta}(t, 0)\right) e_{M}(t, 0) \\
&+M V(x(t)) e_{M}(t, 0) \\
&=\left(-\lambda_{2} V(x(t))+M V(x(t))-L(M \ominus \delta) e_{\ominus \delta}(t, 0)\right) e_{M}(t, 0) \\
& \leq\left(\left(M-\lambda_{2}\right) V(x(t))-L(M \ominus \delta) e_{\ominus \delta}(t, 0)\right) e_{M}(t, 0) \\
& \leq-L(M \ominus \delta) e_{\ominus \delta}(t, 0) e_{M}(t, 0) \\
&=-L(M \ominus \delta) e_{M \ominus \delta}(t, 0) .
\end{aligned}
$$

Integrating both sides of the above inequality from $t_{0}$ to $t$, we obtain, for $t \in\left[t_{k-1}, t_{k}\right)$,

$$
\begin{aligned}
V(x(t)) e_{M}(t, 0) \leq & V\left(x_{0}\right) e_{M}\left(t_{0}, 0\right)-L e_{M \ominus \delta}(t, 0) \\
& +L e_{M \ominus \delta}\left(t_{0}, 0\right) \\
\leq & V\left(x_{0}\right) e_{M}\left(t_{0}, 0\right)+L e_{M \ominus \delta}\left(t_{0}, 0\right) \\
\leq & \left(V\left(x_{0}\right)+L\right) e_{M}\left(t_{0}, 0\right) .
\end{aligned}
$$

This implies that

$$
\begin{aligned}
V(x(t)) & \leq\left(\left(V\left(x_{0}\right)+L\right) e_{M}\left(t_{0}, 0\right)\right) e_{\ominus M}(t, 0) \\
& =\left(V\left(x_{0}\right)+L\right) e_{\ominus M}\left(t, t_{0}\right) .
\end{aligned}
$$

From (26) and by invoking condition (22) we obtain, for all $t \in\left[t_{k-1}, t_{k}\right), k=1,2, \ldots, n$,

$$
\|\psi x(t)\| \leq \lambda_{1}^{-1 / p}\left(\left(V\left(x_{0}\right)+L\right) e_{\ominus M}\left(t, t_{0}\right)\right)^{1 / p} .
$$

By Definition 1 system (1) is $\psi$ uniformly exponentially stable. 
Theorem 6. Assume that $D \subset \mathbb{R}^{n}$ contains the origin and there exists a type I Lyapunov function $V: \mathbb{T}_{t_{0}}^{+} \times D \rightarrow[0, \infty)$ such that, for all $(t, x) \in \mathbb{T}_{t_{0}}^{+} \times D$ and $t \in\left[t_{k-1}, t_{k}\right), k=1,2, \ldots, n$,

$$
\begin{gathered}
\lambda_{1}\|\psi(t) x(t)\|^{p} \leq V(x) \leq \lambda_{2}\|\psi(t) x(t)\|^{p}, \\
V^{\Delta}(t, x) \leq \frac{-\lambda_{3}\|\psi(t) x(t)\|^{p}-L(K \ominus \delta) e_{\ominus \delta}(t, 0)}{1+K \mu(t)},
\end{gathered}
$$

where $\lambda_{1}, \lambda_{2}, \lambda_{3}$, and $p$ are positive constants, $K=\lambda_{3} / \lambda_{2}, L \geq$ $\lambda_{1}$ is a nonnegative constant, and $\delta>\lambda_{3} / \lambda_{2}$. Then the trivial solution to (1) is $\psi$ globally exponentially stable on $[0, \infty)$.

Proof. Let $x$ be a solution to (1) that stays in $D$ for all $t \geq t_{0}$. Since $K=\lambda_{3} / \lambda_{2}, e_{K}(t, 0)$ is well defined and positive. For all $t \in\left[t_{k-1}, t_{k}\right), k=1,2, \ldots, n$, consider

$$
\begin{aligned}
& {\left[V(x(t)) e_{K}(t, 0)\right]^{\Delta} } \\
&=V^{\Delta}(t, x(t)) e_{K}^{\sigma}(t, 0)+V(x(t)) e_{K}^{\Delta}(t, 0) \\
& \leq\left(-\lambda_{3}\|\psi(t) x(t)\|^{p}-L(K \ominus \delta) e_{\ominus \delta}(t, 0)\right) e_{K}(t, 0) \\
&+K V(x(t)) e_{K}(t, 0) \\
&=\left(-\lambda_{3}\|\psi(t) x(t)\|^{p}+K V(x(t))-L(K \ominus \delta) e_{\ominus \delta}(t, 0)\right) \\
& \times e_{K}(t, 0) \\
& \leq\left(\frac{-\lambda_{3}}{\lambda_{2}} V(x(t))+K V(x(t))-L(K \ominus \delta) e_{\ominus \delta}(t, 0)\right) e_{K}(t, 0) \\
&=\left(-L(K \ominus \delta) e_{\ominus \delta}(t, 0)\right) e_{K}(t, 0) \\
&=-L(K \ominus \delta) e_{K \ominus \delta}(t, 0) .
\end{aligned}
$$

Integrating both sides of the above inequality from $t_{0}$ to $t, t \neq t_{k}$, with $x_{0}=x\left(t_{0}\right)$, we obtain,

$$
\begin{aligned}
V(x(t)) e_{K}(t, 0) \leq & V\left(x_{0}\right) e_{K}\left(t_{0}, 0\right) \\
& +L\left(e_{K \ominus \delta}\left(t_{0}, 0\right)-e_{K \ominus \delta}(t, 0)\right) \\
\leq & V\left(x_{0}\right) e_{K}\left(t_{0}, 0\right)+L e_{K \ominus \delta}\left(t_{0}, 0\right) \\
\leq & \left(V\left(x_{0}\right)+L\right) e_{K}\left(t_{0}, 0\right) .
\end{aligned}
$$

This implies that

$$
\begin{aligned}
V(x(t)) & \leq\left(\left(V\left(x_{0}\right)+L\right) e_{K}\left(t_{0}, 0\right)\right) e_{\ominus K}(t, 0) \\
& =\left(V\left(x_{0}\right)+L\right) e_{\ominus K}\left(t, t_{0}\right) .
\end{aligned}
$$

From (32), and by invoking condition (28), we obtain, for all $t \in\left[t_{k-1}, t_{k}\right), k=1,2, \ldots, n$,

$$
\begin{aligned}
\|\psi(t) x(t)\| & \leq \lambda_{1}^{-1 / p}\left(\left(V\left(x_{0}\right)+L\right) e_{\ominus K}\left(t, t_{0}\right)\right)^{1 / p} \\
& \leq \lambda_{1}^{-1 / p}\left(\left(V\left(x_{0}\right)+L\right) e_{\ominus K}\left(t, t_{0}\right)\right)^{1 / p} .
\end{aligned}
$$

If we set $M:=\left(\left(V\left(x_{0}\right)+L\right) / \lambda_{1}\right)^{1 / p}$, then (33) can be written as

$$
\|\psi(t) x(t)\| \leq M\left(e_{\ominus K}\left(t, t_{0}\right)\right)^{1 / p} .
$$

Since $M \geq 1$, by Definition 2 system (1) is $\psi$ globally exponentially stable.

\section{Examples}

Example 7. We consider Example (35) in [7] and extend the example by using impulse condition,

$$
\begin{gathered}
x^{\Delta}=-x+\frac{1}{5} x^{1 / 3} e_{\ominus \delta}(t, 0), \quad t \neq t_{k}, t \in \mathbb{T}, \\
x\left(t_{k}^{+}\right)=-\frac{1}{3}, \quad t=k, k=1,2, \ldots, n,
\end{gathered}
$$

where $\delta>0$ is a constant $x_{0} \in \mathbb{R}$. If there is a constant $0<$ $M<\delta$ such that

$$
\begin{gathered}
(\mu(t)-1)(1+M \mu(t)) \leq-M, \\
\left(\frac{2}{3}\left(\frac{1}{25} \mu(t)\right)^{3 / 2}+\frac{|(2 / 5)-(2 / 5) \mu(t)|^{3}}{3}\right)(1+M \mu(t)) \\
\leq-L(M \ominus \delta)(t),
\end{gathered}
$$

for some constant $L \geq 0$ and all $t \neq k$, (35) is $\psi$ uniformly exponentially stable.

Under above assumptions, we will show that the conditions of Theorem 4 are satisfied. Let $\psi(t)=1 / 2$, choose $D=\mathbb{R}$ and $V(x)=x^{2}, t \neq k$, then (11) holds with $p=q=2, \lambda_{1}=$ $\lambda_{2}=4$. If we calculate $V^{\Delta}$, for all $t \neq k$,

$$
\begin{aligned}
V^{\Delta}= & 2 x\left(-x+\frac{1}{5} x^{1 / 3} e_{\ominus \delta}(t, 0)\right) \\
& +\mu(t)\left(-x+\frac{1}{5} x^{1 / 3} e_{\ominus \delta}(t, 0)\right)^{2},
\end{aligned}
$$

we have the following comparison:

$$
\begin{aligned}
V^{\Delta}= & 2 x\left(-x+\frac{1}{5} x^{1 / 3} e_{\ominus \delta}(t, 0)\right) \\
& +\mu(t)\left(-x+\frac{1}{5} x^{1 / 3} e_{\ominus \delta}(t, 0)\right)^{2} \\
\leq & (\mu(t)-1) x^{2} \\
& +\left[\frac{2}{3}\left(\frac{1}{25} \mu(t)\right)^{3 / 2}+\frac{|(2 / 5)-(2 / 5) \mu(t)|^{3}}{3}\right] e_{\ominus \delta}(t, 0) .
\end{aligned}
$$

Dividing and multiplying the right-hand side by $(1+M \mu(t))$, we see that (12) holds under the above assumptions with $r=2$ and $\lambda_{3}=4 M$. Also, since $p=q=2$, we have

$$
V(x)-V^{r / q}(x)=x^{2}-\left(x^{2}\right)^{2 / 2}=0 \leq \gamma e_{\ominus \delta}\left(t, t_{0}\right),
$$


for all $t \neq k$. Therefore (13) is satisfied. Hence, all hypotheses of Theorem 4 are satisfied and we conclude that the trivial solution to (35) is $\psi$ uniformly exponentially stable. We consider following two special cases of (35).

Case 1. If $\mathbb{T}=\mathbb{R}$, then $\mu(t)=0$. It is easy to see that (37) holds for $M=1$. Also for $L=8 /[375(\delta-M)]$, condition (38) is satisfied. Hence, we conclude that if $\delta>1$, then the trivial solution to (35) is $\psi$ uniformly exponentially stable.

Case 2. If $\mathbb{T}=(1 / 2) \mathbb{Z}$, then $\mu(t)=1 / 2$. In this case rewriting (37) we have

$$
\left(-\frac{1}{2}\right)\left(1+\frac{M}{2}\right) \leq-M,
$$

then (37) holds for $2 / 3>M>0$. Also for $L=((6+\sqrt{2}) /$ $2250(\delta-M))(1-(M / 2))(1-(\delta / 2))$, condition (38) is satisfied. Therefore for $\delta>2 / 3$, then the trivial solution to (35) is $\psi$ uniformly exponentially stable.

\section{References}

[1] V. Lakshmikantham, D. D. Baĭnov, and P. S. Simeonov, Theory of Impulsive Differential Equations, vol. 6 of Series in Modern Applied Mathematics, World Scientific Publishing, Teaneck, NJ, USA, 1989.

[2] D. D. Baĭnov and P. S. Simeonov, Systems with Impulse Effect: Stability, Theory and Applications, Ellis Horwood Series: Mathematics and its Applications, Ellis Horwood, Chichester, UK, 1989.

[3] B. Gupta and S. K. Srivastava, " $\psi$-exponential stability for nonlinear impulsive differential equations," International Journal of Computational and Mathematical Sciences, vol. 4, no. 7, pp. 329333, 2010.

[4] J. Hoffacker and C. C. Tisdell, "Stability and instability for dynamic equations on time scales," Computers \& Mathematics with Applications, vol. 49, no. 9-10, pp. 1327-1334, 2005.

[5] J. J. DaCunha, "Stability for time varying linear dynamic systems on time scales," Journal of Computational and Applied Mathematics, vol. 176, no. 2, pp. 381-410, 2005.

[6] A. C. Peterson and C. C. Tisdell, "Boundedness and uniqueness of solutions to dynamic equations on time scales," Journal of Difference Equations and Applications, vol. 10, no. 13-15, pp. 1295-1306, 2004.

[7] A. C. Peterson and Y. N. Raffoul, "Exponential stability of dynamic equations on time scales," Advances in Difference Equations, vol. 2005, Article ID 858671, 2005.

[8] A.-L. Liu, "Boundedness and exponential stability of solutions to dynamic equations on time scales," Electronic Journal of Differential Equations, vol. 2007, article 12, 14 pages, 2007.

[9] S. K. Choi, N. J. Koo, and D. M. Im, " $h$-stability for linear dynamic equations on time scales," Journal of Mathematical Analysis and Applications, vol. 324, no. 1, pp. 707-720, 2006.

[10] M. Benchohra, J. Henderson, and S. Ntouyas, Impulsive Differential Equations and Inclusions, vol. 2 of Contemporary Mathematics and Its Applications, Hindawi Publishing Corporation, New York, NY, USA, 1st edition, 2006.

[11] M. Benchohra, J. Henderson, S. K. Ntouyas, and A. Ouahab, "On first order impulsive dynamic equations on time scales," Journal of Difference Equations and Applications, vol. 10, no. 6, pp. 541-548, 2004.
[12] V. Lakshmikantham and A. S. Vatsala, "Hybrid systems on time scales," Journal of Computational and Applied Mathematics, vol. 141, no. 1-2, pp. 227-235, 2002.

[13] V. Lupulescu and A. Zada, "Linear impulsive dynamic systems on time scales," Electronic Journal of Qualitative Theory of Differential Equations, no. 11, pp. 1-30, 2010.

[14] E. R. Kaufmann, N. Kosmatov, and Y. N. Raffoul, "Impulsive dynamic equations on a time scale," Electronic Journal of Differential Equations, vol. 2008, article 67, 9 pages, 2008.

[15] Y. Ma and J. Sun, "Stability criteria for impulsive systems on time scales," Journal of Computational and Applied Mathematics, vol. 213, no. 2, pp. 400-407, 2008.

[16] İ. B. Yaşar and A. Tuna, " $\psi$-uniformly stability for time varying linear dynamic systems on time scales," International Mathematical Forum, vol. 2, no. 17-20, pp. 963-972, 2007.

[17] M. Bohner and A. Peterson, Dynamic Equations on Time Scales, An Introduction with Applications, Birkhäuser, Boston, Mass, USA, 2001. 


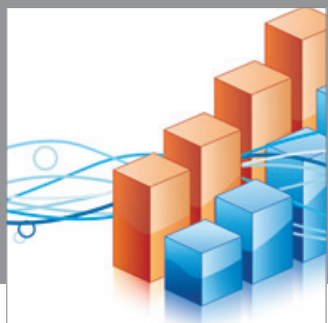

Advances in

Operations Research

mansans

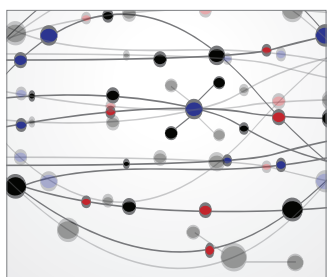

The Scientific World Journal
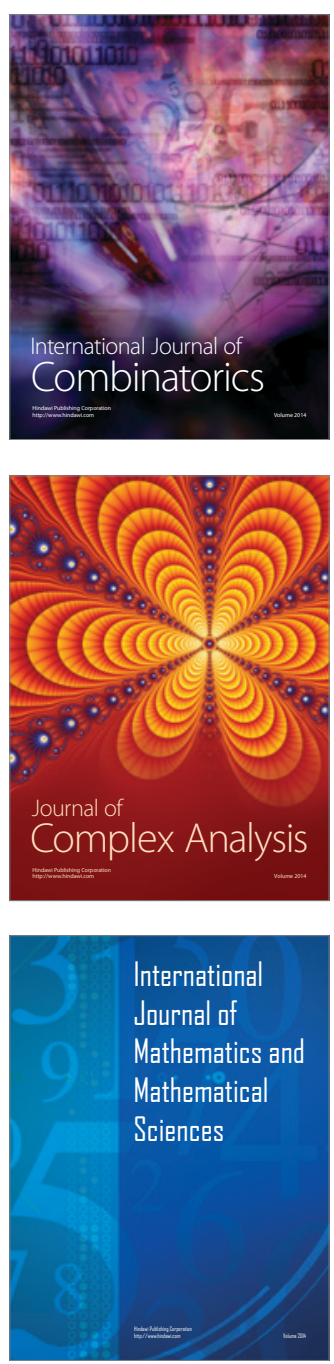
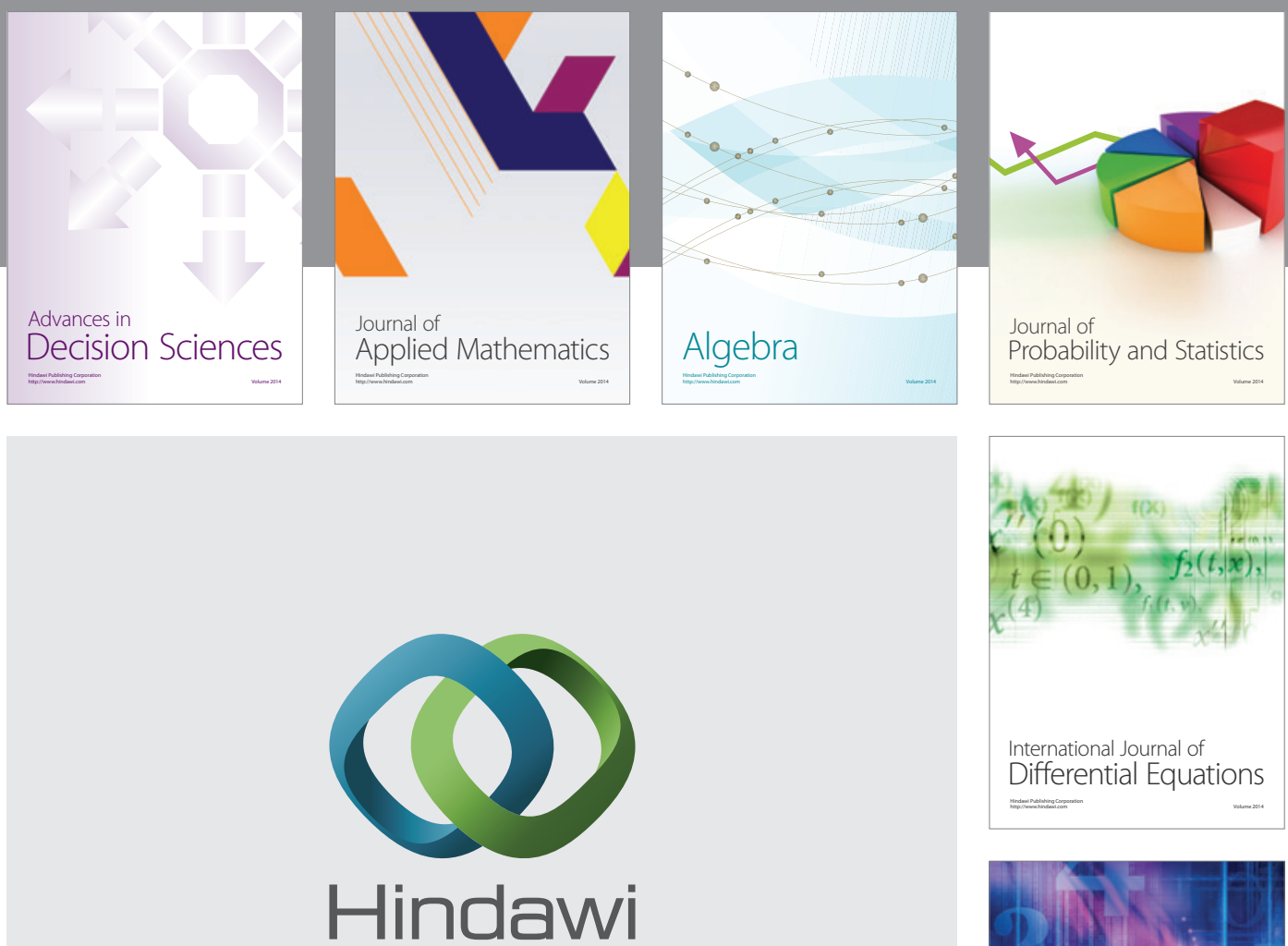

Submit your manuscripts at http://www.hindawi.com
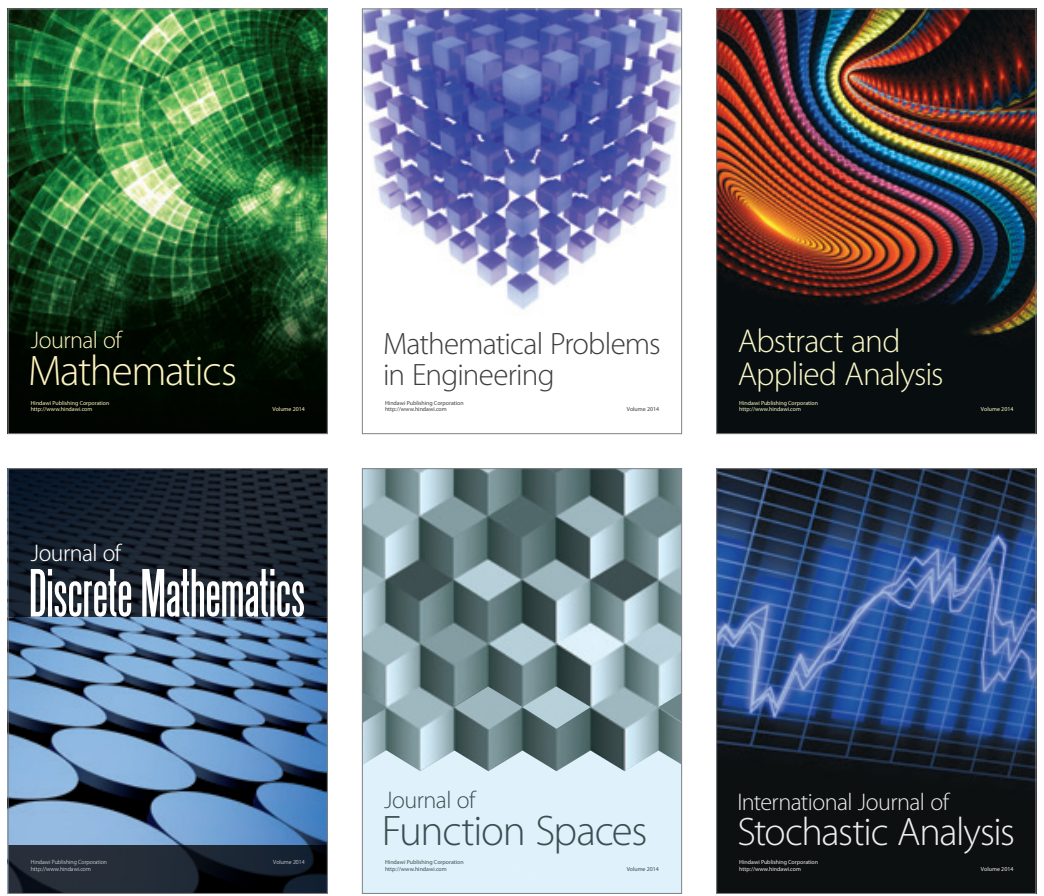

Journal of

Function Spaces

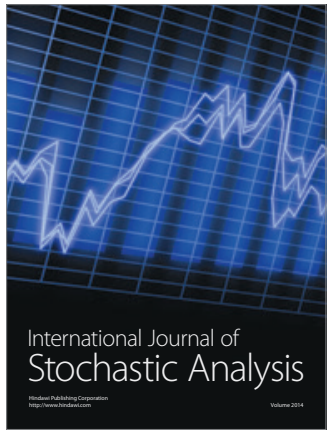

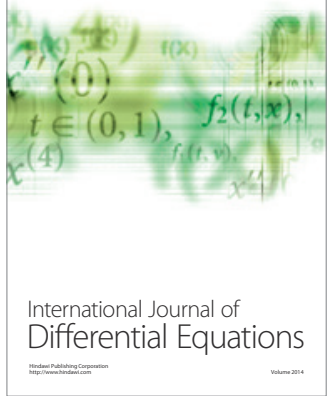
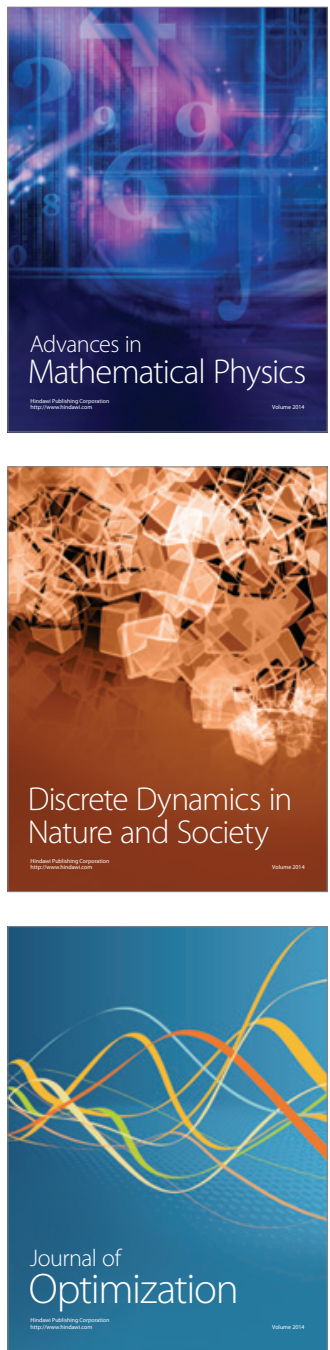